\title{
Genetic Diversity and Antimicrobial Drug Resistance of Serotype VI Group B Streptococcus, Canada
}

\section{Alefiya Neemuchwala, Sarah Teatero, Lindsay Liang, Irene Martin, Walter Demzcuk, Allison McGeer, Nahuel Fittipaldi}

\begin{abstract}
Author affiliations: Public Health Ontario Laboratory, Toronto, Ontario, Canada (A. Neemuchwala, S. Teatero, L. Liang, N. Fittipaldi); National Microbiology Laboratory, Public Health Agency of Canada, Winnipeg, Manitoba, Canada (I. Martin, W. Demzcuk); University of Toronto, Toronto (A. McGeer, N. Fittipaldi); Mount Sinai Hospital, Toronto (A. McGeer)

DOI: https://doi.org/10.3201/eid2410.171711
\end{abstract}

Two genetically dissimilar sequence type 1 clades dominate the serotype VI group B Streptococcus population of strains causing invasive disease in Canada. Isolates of this rare serotype, recovered mainly from adult patients, were all susceptible to penicillin and vancomycin. However, we observed resistance to erythromycin and clindamycin

Serotype VI group B Streptococcus (GBS), which is common in Japan (prevalence rates 16\%-40\%) and has recently emerged in Malaysia and Taiwan, remains rare in Europe and North America (1-4). However, invasive serotype VI infections have been noticed in Alberta and Ontario, Canada $(5,6)$, and unpublished surveillance data for Canada (National Microbiology Laboratory, https://www. canada.ca/en/public-health/services/publications/drugshealth-products/national-laboratory-surveillance-invasivestreptococcal-disease-canada-annual-summary-2015.html) show low frequency $(1.2 \%-4.1 \%)$ but sustained isolation of this GBS serotype in recent years. Here, we characterize a collection of 26 invasive serotype VI GBS strains recovered by passive surveillance in central Canada during 2010-2014 (online Technical Appendix 1, https://wwwnc.cdc.gov/EID/ article/24/10/17-1711-Techapp1.xlsx). Two isolates came from early onset disease (patients age 0-6 days) and 1 from late-onset disease (patients age 7-90 days). Twenty-two isolates came from adult patients ( 9 age 18-60 years and 13 age $>60$ years, a distribution similar to that reported for adult patients with serotype V or serotype IV invasive disease in Canada $[5,6]$ ). Patient age was not available for 1 isolate.

We sequenced the genomes of all isolates using Illumina technology (Illumina, San Diego, CA, USA; National Center for Biotechnology Information BioProject PRJNA420560) and performed in silico multilocus sequence typing. Isolates belonged to sequence types (STs) ST889 (n $=1)$, ST297 $(n=1)$, ST14 $(n=2)$, and ST1 $(n=22)$ (online Technical Appendix 1). ST297, ST14, and ST1 are members of clonal complex (CC) 1; most serotype IV and V isolates responsible for adult disease in Canada also belong to $\mathrm{CC} 1$ (5-7). However, genome-wide, single-nucleotide polymorphism (SNP)-based phylogenetic analysis showed that $\mathrm{CC} 1$ isolates of these 3 serotypes are genetically dissimilar (online Technical Appendix 2 Figure 1, https:// wwwnc.cdc.gov/EID/article/24/10/17-1711-Techapp2.pdf; genome-wide SNPs were identified relative to the genome of GBS-M002, a serotype VI isolate from Taiwan [GenBank accession no. CP013908.1]). Antimicrobial drug resistance among serotype VI isolates was, overall, similar to that described among serotype IV and V isolates causing adult invasive disease in Canada $(5,8)$ (MICs for penicillin, erythromycin, clindamycin, tetracycline, and vancomycin were determined using the agar dilution method or Etest according to Clinical and Laboratory Standards Institute guidelines [9]). All serotype VI isolates were susceptible to penicillin and vancomycin (online Technical Appendix 1). Resistance to erythromycin was found in $10(38 \%)$ invasive isolates, resistance to clindamycin in $9(35 \%)$, and resistance to tetracycline in $8(31 \%)$ (online Technical Appendix 1). All lincosamide- and macrolide-resistant strains possessed gene ermB; 1 isolate had genes mefA and $m s r D$. Genes tet $S$, tet $M$, and tet $O$ were associated with observed resistance to tetracycline (online Technical Appendix 1).

Most $(\mathrm{n}=22)$ ST1 isolates in our collection had a pilus island (PI) profile consisting of PI-1 containing the recently described PI-1 backbone protein subunit BP$1 \mathrm{~b}$ (10) (BP1b-PI-1), in combination with PI-2a (online Technical Appendix 1). One ST1 isolate (NGBS1605) possessed the traditional PI-1 and PI-2a (online Technical Appendix 1). The ST14 isolates had BP1b-PI-1 and PI-2b. The ST889 isolate possessed only PI-2a (online Technical Appendix 1). We found differences among isolates in genes encoding $\alpha$-like proteins (Alps): the ST297 isolate and most ST1 strains had gene bca encoding $\alpha$-C protein. ST1 isolates NGBS543 and NGBS1605 possessed gene alp3, encoding Alp3. The ST14 and ST889 isolates possessed gene alp1, encoding Alp1 (or epsilon) protein (online Technical Appendix 1).

We next examined the extent of genetic diversity among the numerically dominant group of serotype VI ST1 organisms. For comparative purposes, genome data for 3 additional serotype VI strains were included (French strain CCH330, SRA accession no. ERX298473; Malaysian strain PR06, GenBank accession no. AOSD00000000.1; and 1 temporally matched serotype VI isolate recovered from a colonized pregnant woman in Canada; online Technical Appendix 1). Recombination was the main driver of genetic diversity among serotype VI ST1 organisms. Most $(n=16)$ 
ST1 isolates clustered closely with Malaysia strain PR06 (online Technical Appendix 2 Figure 2). This clade (arbitrarily named the Malaysian clade) included most ST1 isolates with resistance to erythromycin and clindamycin. Recombination in a region of $\approx 200 \mathrm{kbp}$ containing the genes encoding the 2-component virulence regulator CsrRS differentiated the Malaysian clade from a second clade formed by 5 Canadian isolates and the French and Taiwanese ST1 isolates (arbitrarily named the Taiwanese clade) (online Technical Appendix 2 Figure 2). Recombination also explains the aforementioned differences in Alp- and pilus subunit-encoding genes among serotype VI ST1 strains. Isolates NGBS543 and NGBS1605 differed from other ST1 isolates by recombination in a region spanning 107 and 89 $\mathrm{kbp}$, respectively, containing Alp-encoding genes. These 2 isolates also differed between themselves by recombination in the PI-1 locus (online Technical Appendix 2 Figure 2).

Global travel and migration are known contributors to the emergence of bacterial clones in new geographies (11). Serotype VI GBS infections have emerged in Malaysia and Taiwan $(3,4)$. The population of serotype VI GBS isolates in Canada is dominated by 2 ST1 clades, each closely related genetically to the Malaysian or Taiwanese isolates. Although it is tempting to speculate that these 2 ST1 genotypes were introduced into Canada from overseas, the speculation cannot be fully supported by our current limited dataset. Continued monitoring for serotype VI GBS infections is warranted.

\section{Acknowledgments}

We thank staff at Public Health Ontario Genome Core for genome sequencing of our strains.

This work was supported by Public Health Ontario through internal grant RRB-17-030 to N.F.

\section{About the Author}

Ms. Neemuchwala is a research associate at Public Health Ontario in Toronto, Ontario, Canada. Her research interests include the molecular epidemiology of pathogenic streptococci.

\section{References}

1. Le Doare K, Heath PT. An overview of global GBS epidemiology. Vaccine. 2013;31(Suppl 4):D7-12. http://dx.doi.org/10.1016/ j.vaccine.2013.01.009

2. Morozumi M, Wajima T, Takata M, Iwata S, Ubukata K. Molecular characteristics of group B Streptococci isolated from adults with invasive infections in Japan. J Clin Microbiol. 2016;54:2695-700. http://dx.doi.org/10.1128/JCM.01183-16

3. Eskandarian N, Ismail Z, Neela V, van Belkum A, Desa MN, Amin Nordin S. Antimicrobial susceptibility profiles, serotype distribution and virulence determinants among invasive, non-invasive and colonizing Streptococcus agalactiae (group B Streptococcus) from Malaysian patients. Eur J Clin Microbiol Infect Dis. 2015;34:579-84. http://dx.doi.org/10.1007/s10096-014-2265-x

4. Lin HC, Chen CJ, Chiang KH, Yen TY, Ho CM, Hwang KP, et al. Clonal dissemination of invasive and colonizing clonal complex 1 of serotype VI group B Streptococcus in central Taiwan. J Microbiol Immunol Infect. 2016;49:902-9. http://dx.doi.org/ 10.1016/j.jmii.2014.11.002

5. Alhhazmi A, Hurteau D, Tyrrell GJ. Epidemiology of invasive group B streptococcal disease in Alberta, Canada, from 2003 to 2013. J Clin Microbiol. 2016;54:1774-81. http://dx.doi.org/ 10.1128/JCM.00355-16

6. Teatero S, McGeer A, Low DE, Li A, Demczuk W, Martin I, et al. Characterization of invasive group B Streptococcus strains from the greater Toronto area, Canada. J Clin Microbiol. 2014;52:1441-7. http://dx.doi.org/10.1128/JCM.03554-13

7. Flores AR, Galloway-Peña J, Sahasrabhojane P, Saldaña M, Yao H, $\mathrm{Su} \mathrm{X}$, et al. Sequence type 1 group B Streptococcus, an emerging cause of invasive disease in adults, evolves by small genetic changes. Proc Natl Acad Sci U S A. 2015;112:6431-6. http://dx.doi.org/10.1073/pnas.1504725112

8. Teatero S, Athey TB, Van Caeseele P, Horsman G, Alexander DC, Melano RG, et al. Emergence of serotype IV group B Streptococcus adult invasive disease in Manitoba and Saskatchewan, Canada, is driven by clonal sequence type 459 strains. J Clin Microbiol. 2015;53:2919-26. http://dx.doi.org/10.1128/JCM.01128-15

9. Clinical and Laboratory Standards Institute. Performance standards for antimicrobial susceptibility testing. 25th informational supplement (M100-S23). Wayne (PA): The Institute; 2015.

10. Teatero S, Neemuchwala A, Yang K, Gomes J, Athey TBT, Martin I, et al. Genetic evidence for a novel variant of the pilus island 1 backbone protein in group B Streptococcus. J Med Microbiol. 2017;66:1409-15. http://dx.doi.org/10.1099/jmm.0.000588

11. Morens DM, Fauci AS. Emerging infectious diseases: threats to human health and global stability. PLoS Pathog. 2013;9:e1003467. http://dx.doi.org/10.1371/journal.ppat.1003467

Address for correspondence: Nahuel Fittipaldi, Public Health Ontario Laboratory, 661 University Ave, Ste 17-100, Toronto, ON M5G 1M1, Canada; email: nahuel.fittipaldi@oahpp.ca

\section{Psychrobacter sanguinis Wound Infection Associated with Marine Environment Exposure, Washington, USA}

\author{
Jesse Bonwitt, Michael Tran, Angela Droz, \\ Anna Gonzalez, William A. Glover
}

Author affiliations: Centers for Disease Control and Prevention, Atlanta, Georgia, USA (J. Bonwitt); Durham University, Durham, England (J. Bonwitt); Washington State Department of Health, Shoreline, Washington, USA (J. Bonwitt, M. Tran, W.A. Glover); Harrison Medical Center, Bainbridge Island, Washington, USA (A. Droz); Kitsap Public Health District, Bremerton, Washington, USA (A. Gonzalez)

DOI: https://doi.org/10.3201/eid2410.171821 